\title{
Pérdida de bosque en zonas para la conservación y recuperación de la Zonificación Ecológica y Económica (ZEE) de la provincia Alto Amazonas, Loreto, Perú
}

\author{
[Forest loss in areas of conservation and recovery of the Ecological and \\ Economic Zoning (ZEE) in the province of Alto Amazonas, Loreto, Peru]
}

\author{
Juan José Palacios-Vega ${ }^{1 *}$, Ricardo Zárate-Gómez ${ }^{1}$, Manuel Martín-Brañas ${ }^{1}$, Reynaldo Minaya-Vela² \\ 1. Instituto de Investigaciones de la Amazonía Peruana (IIAP). Dirección de Investigación en Sociedades Amazónicas \\ (SOCIODIVERSIDAD). Av. Quiñones km 2,5, San Juan Bautista, Maynas, Loreto, Perú. \\ Correos electrónicos: jpalacios@iiap.gob.pe (J. J. Palacios-Vega * Autor para correspondencia), \\ rzarate@iiap.gob.pe (R. Zárate-Gómez), mmartin@iiap.gob.pe (M. Martín-Brañas). \\ 2. Gobierno Regional de Loreto. Av. Abelardo Quiñones km 1,5, Belén, Maynas, Loreto, Perú. \\ Correo electrónico: reynaldo.minayav@gmail.com (R. J. Minaya-Vela).
}

\section{Resumen}

En el año 2015 se aprobó la Zonificación Ecológica Económica (ZEE) de la provincia Alto Amazonas (PAA) Loreto, Perú; para su gestión territorial adecuada. Sin embargo, la pérdida de cobertura boscosa sigue en aumento. En este sentido, el objetivo del presente estudio fue analizar los patrones espaciales por la pérdida del bosque en la PAA en el periodo 2012-2019. Se calculó el índice de pérdida acumulada de bosque (IPB) y se analizaron los patrones espaciales originados por la pérdida del bosque mediante el índice de autocorrelación espacial local de Getis-Ord (G*). Se generaron mapas de distribución del IPB y se identificaron patrones de agrupamiento de puntos calientes y fríos desde una significancia $p<0,05$. La agrupación de puntos calientes se distribuye principalmente al sur de la PAA y los puntos fríos se distribuyen al centro y norte de la PAA. En zonas propuestas para la protección y conservación ecológica la pérdida del bosque se representa en patrones espaciales de puntos fríos, y en zonas para recuperación en patrones de puntos calientes. La presente información permite evidenciar un inadecuado uso de la información de la ZEE en los procesos de toma de decisiones sobre la gestión del territorio.

Palabras clave: Amazonía Peruana, Deforestación, Análisis Espacial, Ordenamiento Territorial, Sistema de Información Geográfica (SIG).

\section{Abstract}

In 2015 the Ecological Economic Zoning (ZEE) was approved in the province of Alto Amazonas (PAA) Loreto. The ZEE was instated to properly organize national territory. However, the loss of forest cover continues to increase. In this sense, the objective of the present study was to analyze the spatial patterns by forest loss in the PAA in the period 2012-2019. The cumulative forest loss index (IPB) was calculated and spatial patterns resulting from the loss of the forest were analyzed by local spatial autocorrelation index Getis-Ord (G*). IPB distribution maps were generated and clustering patterns of hot and cold spots were identified from significance $p<0,05$. The clustering of hot spots is distributed mainly to the south of the PAA and cold spots are distributed to the center and north of the PAA. In areas proposed for ecological protection and conservation, forest loss is represented in spatial patterns of cold spots, and in areas for recovery is in patterns of hot spots. This information shows an inadequate use of ZEE information in the decision-making processes for land management.

Keywords: Deforestation, Geographic Information System, Land Use Planning, Peruvian Amazon, Spatial Analysis. 


\section{INTRODUCCIÓN}

Los instrumentos de gestión, ordenamiento y planificación del territorio en el Perú se remiten a la Zonificación Ecológica Económica (ZEE). La ZEE "es un proceso dinámico y flexible para la identificación de diferentes alternativas de uso sostenible de un territorio determinado, basado en la evaluación de sus potencialidades y limitaciones con criterios físicos, biológicos, sociales, económicos y culturales" (Decreto Supremo $\mathrm{N}^{\circ}$ 087-2004-PCM); siendo un requisito para el diagnóstico del territorio $y$, por tanto, para determinar la focalización de la inversión pública o privada en los usos del territorio (Ballón et al., 2015). Para el departamento Loreto se han realizado al menos 18 estudios de ZEE, como la macrozonificación del Abanico del Pastaza (WWF-IIAP, 2002), mesozonificación del área de influencia de la carretera Iquitos-Nauta (IIAP-AECI-CTARL, 2001), zonificación ecológica y económica de la cuenca Pastaza-Morona (IIAP- MPDM, 2009), entre otros.

La ZEE de la provincia Alto Amazonas, fue la primera que contó con la aprobación del Ministerio del Ambiente (IIAP-GOREL-MPAM, 2015); en ella se proponen diversos tipos de usos del territorio. Sin embargo, en muchos casos las recomendaciones propuestas en este tipo de instrumentos de gestión del territorio no se utilizan o aplican, siendo la ausencia de planes de ordenamiento territorial una de las principales razones para esta falta de aplicación. Esto ha provocado que no se esté utilizando el territorio de acuerdo a sus potencialidades y limitaciones, y se continué deforestando (pérdida de cobertura del bosque) en aquellas zonas recomendadas para la conservación y protección (Mengatto et al., 2017).

La pérdida de cobertura del bosque es el cambio de la cobertura vegetal originado por la acción antrópica o natural, se da principalmente por la deforestación para expansión agrícola y actividades extractivas ilegales o informales, así como, de forma natural, debido a vendavales o deslizamientos de tierra (Vargas et al., 2019).
La pérdida del bosque implica grandes transformaciones del territorio y del paisaje natural; si es comparada o relacionada con otras áreas funcionales, conlleva la modificación y afectación de los espacios geográficos, reflejándose estas relaciones o efectos en patrones espaciales (Otavo y Echevarria, 2017).

La carencia de información sobre la dinámica del bosque y su afectación en los principales ejes de planificación territorial, como la conservación, producción, entre otros; condiciona la toma de decisiones, debido al desconocimiento de información, herramientas, políticas, acciones o estrategias que se deben aplicar en la planificación integral del territorio para poder llegar a una gestión eficaz del mismo y así evitar los conflictos sobre el territorio. Ante esto, es necesario anticipar e identificar potenciales conflictos del territorio mediante la conceptualización y operacionalización de modelos que identifiquen y mapeen índices derivados de valores históricos (Brown y Raymond, 2014). Los datos espaciales de pérdida del bosque, derivados de datos históricos de cambio en la cobertura boscosa, son muy bien procesados en plataformas SIG (Philippe y Karume, 2019).

Las herramientas para el análisis espacial permiten explorar las características estructurales espaciales y la regulación de agrupamientos de pérdida de bosque en unidades espaciales de análisis (Wei et al., 2014). Para identificar y evaluar los patrones espaciales se requiere encontrar formas de modelar las relaciones espaciales, medir la autocorrelación espacial y comprender el fenómeno en cuestión, así como sus procesos espaciales (Siabato y Guzmán, 2019). La identificación y evaluación de un fenómeno se pueden medir y representar en índices combinados con mapas a detalle, generados en modelos utilizando herramientas de análisis espacial y a través de la detección de patrones espaciales como los puntos fríos y calientes (Roces et al., 2016).

Los fenómenos geográficos como el impacto de la pérdida del bosque y su productividad pueden ser cuantificables y medibles en el tiem- 
po mediante la autocorrelación espacial, esta puede ser de tipo global y local (de Moura y Procopiuck, 2020; Ren et al., 2020). A los índices globales se les denomina comúnmente indicadores locales de autocorrelación espacial (LISA); entre ellos encontramos el Índice de Getis-Ord (G*), que se utiliza para analizar e identificar tendencias de agrupaciones de un determinado fenómeno, que pueden ser representadas en zonas de puntos calientes y fríos o en sin significancia (Ren et al., 2020, Zúñiga et al., 2019, Elfadaly et al., 2018; Zúñiga y Pompa, 2018, Ord y Getis, 1995).

La información acerca de la distribución de los patrones espaciales que la pérdida del bosque ha generado sobre las zonas ecológicas económicas propuestas en la PAA, coadyuvaría a aumentar el conocimiento acerca de la distribución espacial de los efectos producidos por el cambio de cobertura y uso de la tierra, para una mejor gestión del territorio. El objetivo del presente estudio fue analizar los patrones espaciales debido a la pérdida de bosque en las zonas destinadas para la conservación y recuperación ecológica durante el periodo 2012-2019, en la provincia Alto Amazonas, Loreto, Perú.

El presente estudio plantea la hipótesis de que los patrones espaciales de puntos calientes debido a una mayor pérdida del bosque se distribuyen en zonas ecológicas recomendadas para la protección y conservación ecológica, y en zonas para la recuperación por el uso inadecuado de la tierra de la Zonificación Ecológica y Económica de la provincia Alto Amazonas. En este sentido se formula la siguiente pregunta de investigación ¿Cómo se distribuyen los patrones espaciales de la pérdida del bosque en las Zonas Ecológicas Económicas de la provincia Alto Amazonas?

\section{MATERIAL Y MÉTODOS}

\section{Área de estudio}

Alto Amazonas es una provincia del departamento Loreto (Perú), su capital es la ciudad de
Yurimaguas, comprende una división político administrativa de seis distritos (Balsapuerto, Jeberos, Lagunas, Santa Cruz, Teniente César López Rojas y Yurimaguas), está conformada por 397 centros poblados y se encuentra enmarcada entre las coordenadas del Sistema Geográfico Mundial (-76,888940, -3,680523; $-75,379596,-6,180441)$. Limita al Norte con las provincias Datem del Marañón y Loreto, al Sur con el departamento San Martín, al Este con las provincias Loreto y Requena del departamento Loreto, y al Oeste con la provincia Datem del Marañón (departamento Loreto) y el departamento San Martín (Figura 1). Comprende una superficie de 2004 287,82 ha y una población de 120480 habitantes. Su rango altitudinal varía desde los 70 a los 2300 metros sobre el nivel del mar. La red hidrográfica que recorre Alto Amazonas corresponde a los ríos Marañón con sus afluentes del norte Nucurai y Pavayacu; desde el sur el río Huallaga con sus afluentes Shanusi, Cachiyacu, Paranapura y Aypena. El clima de Alto Amazonas es semi húmedo cálido, caracterizado por una temperatura media anual que va desde los $22^{\circ} \mathrm{C}$ a los $28^{\circ} \mathrm{C}$, la precipitación promedio anual es de 1967 mm, el mes más lluvioso es marzo, con $275 \mathrm{~mm}$ y el menos lluvioso es setiembre, con $75 \mathrm{~mm}$; la humedad relativa media es de $88 \%$ (Paredes, 2015). El relieve se caracteriza principalmente por dos provincias fisiográficas, la Cordillera andina y subandina con un relieve montañoso muy disectado y la cuenca de sedimentación del Amazonas con un relieve plano y colinoso donde predominan las terrazas medias y las colinas bajas (Escobedo et al., 2015). La vegetación es de tipo inundable y no inundable (tierra firme), conformada por 15 comunidades vegetales, una composición florística de por lo menos 396 especies de Angiospermas, Gimnospermas y Pteridofitas (helechos), incluidas en 225 géneros y 80 familias botánicas (Zárate et al., 2016). Así también, posee una configuración socio cultural compleja con patrones yuxtapuestos en un mismo espacio, los cuales se definen en los frentes sociocultural indígena amazónico, mestizo ribereño, de colonos migrantes y el urbano. La población indígena representa el 20,01 \% de la población total y está conformada por 115 comunidades 


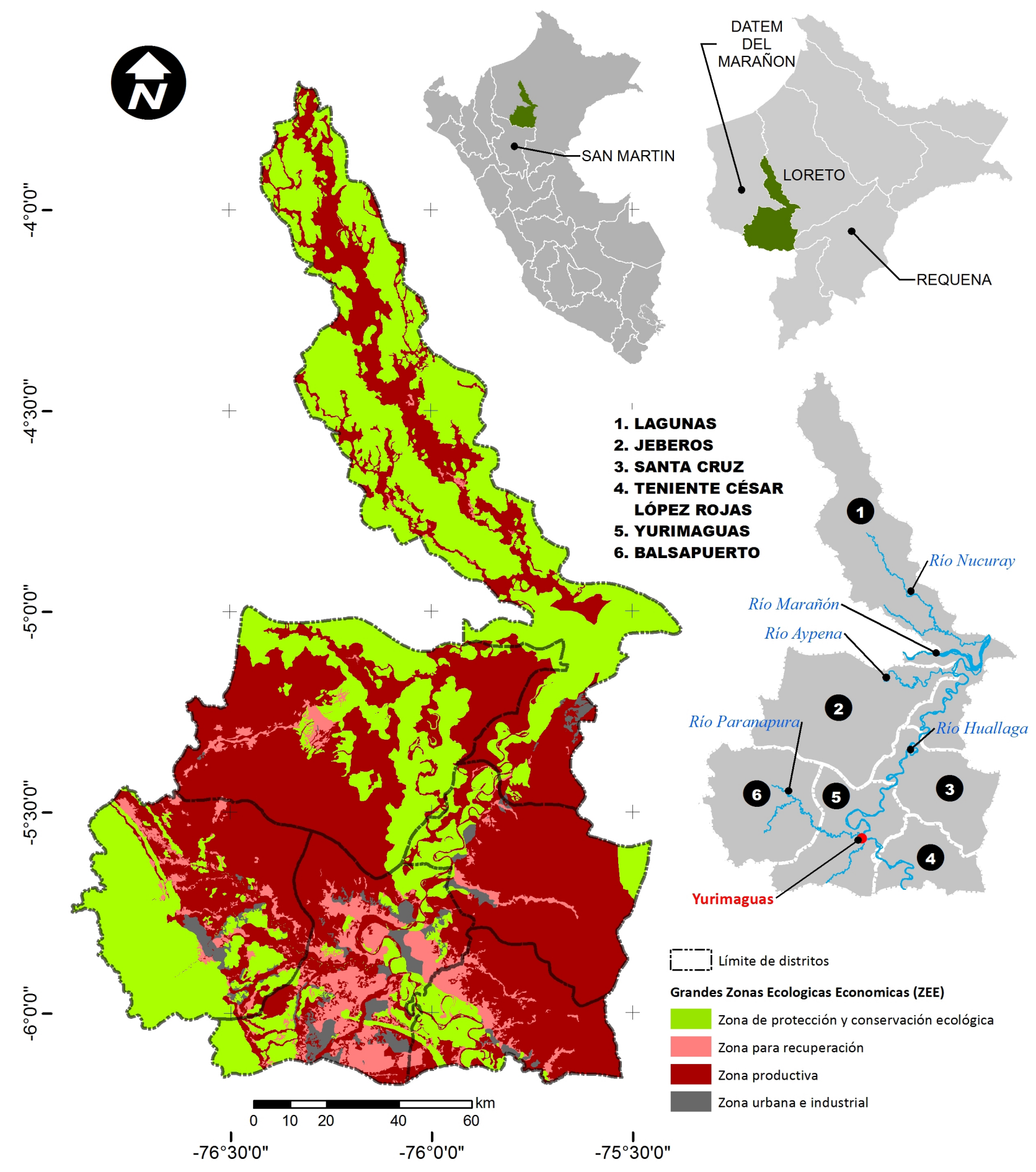

Figura 1. Mapa de ubicación del área de estudio y grandes zonas ecológicas económicas de la provincia Alto Amazonas, Loreto, Perú. 
pertenecientes a nueve pueblos indígenas, entre los cuales figuran los shawi, achuar, chapra, kukama-kukamiria, awuajun, kichwa, kandozi, shiwilu y chamicuro (Álvarez y Jarama, 2015; Limachi y Grandez, 2015; MINCUL, 2021). Es la provincia del departamento Loreto con mayor dinamismo económico debido a su situación estratégica y su conectividad por vía terrestre con otras partes del Perú; el desarrollo urbano es movido por el comercio, las finanzas, el sector administrativo y el transporte (terrestre, aéreo y fluvial), mientras que el desarrollo rural es movido por la agricultura de subsistencia y de monocultivos (palma aceitera) principalmente al sur de la provincia (Álvarez y Jarama, 2015; Limachi y Grandez, 2015). Ha tenido fuertes intervenciones económicas, principalmente de tipo agrícola, a partir del asentamiento de grandes empresas del rubro, así como, la migración de personas naturales de San Martín, Amazonas o Cajamarca dedicados a esta actividad económica. Entre las principales cosechas que producen y comercializan están el camu-camu (Myrciaria dubia), cacao (Theobroma cacao) y palma aceitera (Elaeis guineensis). Cuenta con una Población Económicamente Activa (PEA) de 33372 habitantes y se desarrollan principalmente actividades primarias o extractivas, lo que la convierte en la segunda economía de Loreto, después de Iquitos, la capital departamental (Ocampo et al., 2018).

\section{Fuentes de datos}

Los datos utilizados corresponden a las capas de información vectorial de la propuesta de la ZEE de la PAA del año 2015, que cuenta con opinión favorable del Ministerio del Ambiente (MINAM) y fue generada por el Instituto de Investigaciones de la Amazonía Peruana (IIAP) y el Gobierno Regional de Loreto (GORE Loreto). Estos datos son públicos y de libre descarga desde https://geoservidor.minam.gob.pe/zee-aprobadas/alto-amazonas/. También se recopilaron capas de información raster de las pérdidas de cobertura boscosas, generadas por el Programa Nacional de Conservación de los Bosques del MINAM al año 2019 y descargados desde http://geobosques.minam.gob.pe/geobosque/ view/descargas.php. Todos los datos geoespa- ciales recopilados y generados estuvieron en el Sistema de Referencia WGS 84 y Sistema de Proyección Cartográfico UTM Zona 18 Sur. Para la visualización de las capas de información y migración de vector a raster se utilizó el programa QGIS 3.10, y para el análisis espacial y cálculo del estadístico de Getis-Ord (G*) se utilizó GeoDa 1.16 versión de escritorio para Windows, el cual es un programa de estadística espacial gratuito, de código abierto completo, multiplataforma y de libre descarga en https://geodacenter.github.io/download.html (Anselin, 2021, Anselin et al., 2010).

\section{Cálculo del índice de pérdida de bos- que (IPB)}

Se definió el periodo de análisis extrayendo los datos raster de la pérdida del bosque en el periodo 2012-2019, debido a que la propuesta y mapa de ZEE de la PAA fueron generados con información base geoespacial correspondiente al año 2011. La unidad de análisis espacial fue definida como cada polígono (con identificador único) que conforma cada una de las cuatro grandes zonas ecológicas económicas. Se extrajeron los valores de superficie en hectáreas de pérdida de bosque para cada unidad de análisis. Se calculó el IPB, que varía entre 0 y 1 y se define como el porcentaje de tamaño de parche de pérdida de bosque contenido en cada polígono de unidad de análisis que conforma la ZEE, en donde los valores altos indican que la superficie del parche de pérdida del bosque tiende a ser del mismo tamaño que la unidad de análisis que lo contiene y los valores bajos un tamaño de parche de pérdida de bosque menor.

\section{Evaluación de la pérdida de bosque en la ZEE}

Con el propósito de representar y visualizar la distribución de la pérdida del bosque en el periodo 2012-2019 y su afectación en las unidades de ZEE, se aplicó un mapeo condicionado con valores de IPB clasificados en cuartiles y las cuatro clases de ZEE: Zona productiva (ZP), Zona de protección y conservación ecológica (ZPCE), Zona para recuperación (ZR) y Zona urbana e industrial (ZUI), utilizando el programa GeoDa versión 1.16. 


\section{Análisis de patrones espaciales}

Se utilizó el estadístico de G* propuesto por Getis y Ord (1992) para identificar y analizar la distribución espacial de áreas clave de agrupamientos en puntos calientes y fríos, e indicadores de patrones emergentes del impacto de la pérdida de bosque en cuatro grandes zonas ecológicas y económicas. G* se calcula con la siguiente formula:

$$
G_{i}^{*}(d)=\sum_{j=1}^{n} W_{i j}(d) x_{j} / \sum_{j=1}^{n} x_{j}
$$

Donde $x_{j}$ es el valor del IPB en cada unidad de análisis $j, W_{i j}$ es la matriz de ponderación espacial y $n$ es el total de unidades de análisis. G* se encuentra implementado en GeoDa, en donde se genera una representación de las unidades de análisis con sus valores y de las unidades vecinas comparados con la suma de valores de todas las unidades análisis del área de estudio. G* se estandariza para una mejor interpretación mediante:

$$
Z\left(G_{i}^{*}\right)=\frac{G_{i}^{*}-E\left(G_{i}^{*}\right)}{\sqrt{\operatorname{Var}\left(G_{i}^{*}\right)}}
$$

Donde $E\left(G_{i}^{*}\right)$ y $\operatorname{Var}\left(G_{i}^{*}\right)$ son la expectativa matemática y el coeficiente variable de $\mathrm{G}_{i}{ }^{*}$, respectivamente. La nueva representación contiene $z$-score, $p$-value y el nivel de significancia estadística, G* se interpreta como unidades con valores de $z$-score altos y $p$-value pequeños son indicadores de agrupamiento de puntos calientes estadísticamente significativos, mientras que $z$-score negativo o bajo y $p$-value pequeño indican agrupamiento de puntos fríos estadísticamente significativos al 99\%, 95\% y $90 \%$ ( $\mathrm{Li}$ et al., 2017; Xu et al., 2019; Alvarado et al., 2019; Peng et al., 2019).

\section{RESULTADOS Y DISCUSIÓN}

\section{Distribución del índice de pérdida acu- mulada de bosque (IPB) general $y$ evaluación en la ZEE}

El IPB varía entre de 0 a 0,993, el 50\% de las unidades analizadas concentran una pérdida de bosque aproximada de $20 \%$ durante el periodo 2012-2019. Se identificaron 22 valores atípicos superiores del IPB representados en el mapa condicional de la Figura 2, estos valores empiezan a identificarse a partir del IPB 0,781, esto utilizando un múltiplo estándar de 3 veces el rango intercuartílico (valor de corte). Este múltiplo estándar es muy utilizado para determinar valores extremos y valores atípicos, lo que permite representarlos de mejor forma (Anselin et al., 2010). El 50\% de las unidades de análisis correspondiente a 824 polígonos tienen valores menores del IPB los cuales varían entre 0 y 0,026 . Las otras unidades se distribuyen entre los cuartiles 3 y 4 con 412 y 390 polígonos respectivamente ubicándose principalmente al sureste de la PAA.

Las grandes zonas de la ZEE con mayor IPB son las destinadas para ZR y ZUI, siendo esta última la de mayor variabilidad de IPB, lo que indica que las mayores áreas de pérdida de bosque se concentran en las zonas de recuperación, espacialmente se puede apreciar que más del $50 \%$ de la cantidad de unidades de ZR y ZUI han sido afectas por la pérdida del bosque (Figura 3). Las ZP concentran valores de IPB bajos, lo que implica áreas pequeñas de pérdida de bosque en las unidades de ZP. Las ZPCE presentan una concentración de valores muy menores de IPB, aquí la pérdida de bosque se ha dado en parches de menor superficie (Figura 3).

\section{Análisis de patrones espaciales}

Con la estadística local de G* se obtuvieron agrupamientos de puntos calientes y fríos estadísticamente significativos con niveles de confianza de $90 \%$ a $99 \%(p<0,01)$. El estadístico de Getis-Ord (G*) permitió observar que en la PAA los patrones espaciales de agrupamiento de puntos calientes y fríos del IPB se agrupan 

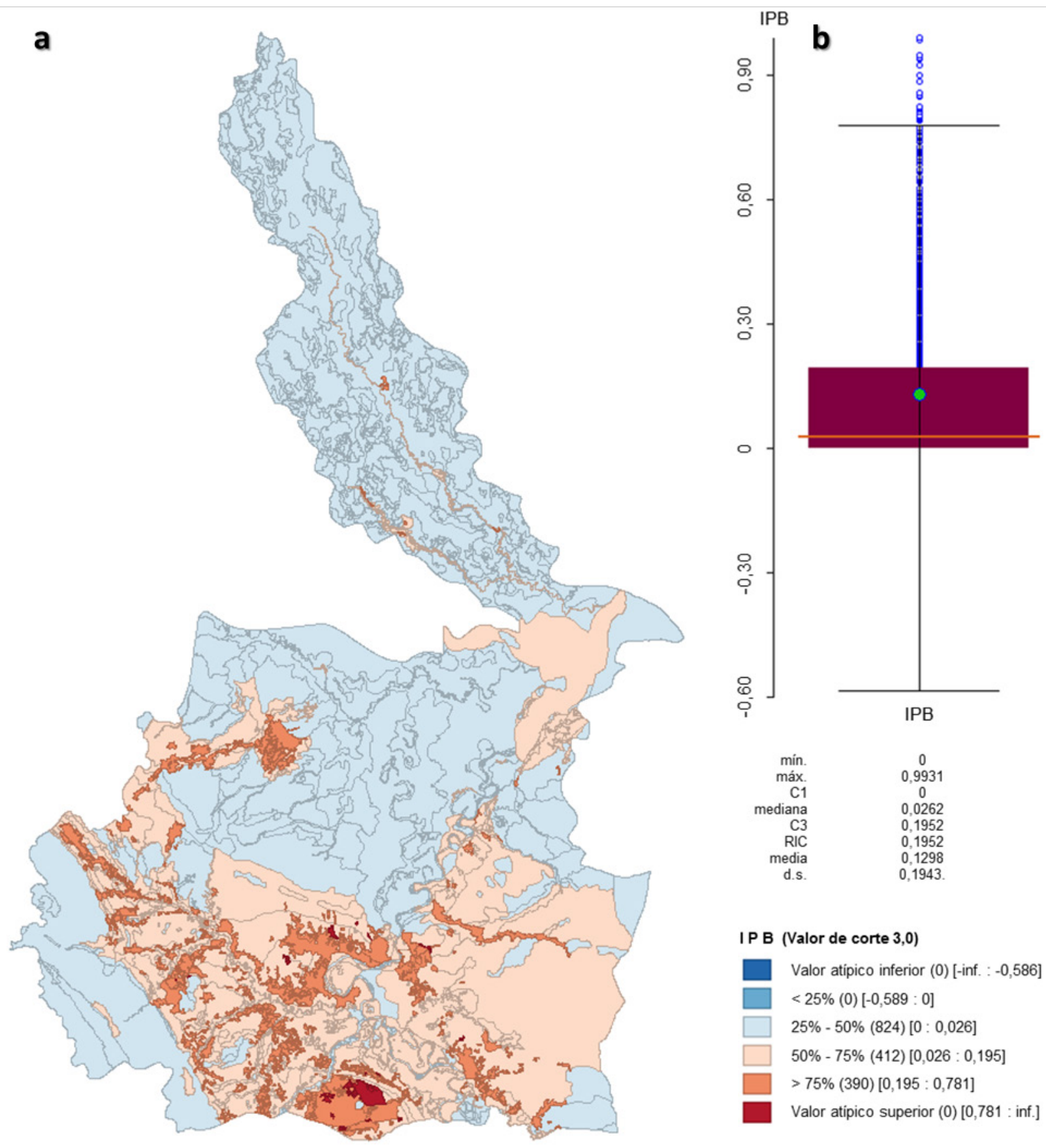

Figura 2. Distribución del índice de pérdida del bosque (IPB) en la provincia Alto Amazonas, departamento Loreto, Perú. a) Diagrama de cajas y bigotes b) Mapa de caja de la distribución del IPB en la PAA, con un valor de corte "Hinge" de 3,0. Elaborado en Geoda 1.16

en superficies de 329200,67 ha que representan el $16,42 \%$ y 301309,42 ha $(15,03 \%)$ respectivamente (Tabla 1).

El grado de agrupación de la pérdida del bosque en el periodo 2012 al 2019, representado en patrones espaciales de puntos calientes, se puede apreciar al sur de la PAA, por otro lado el patrón espacial de puntos fríos se distribuye desde el centro hacia el norte de la PAA (Figura 4). Las agrupaciones de puntos calientes y fríos advierten que el IPB se distribuye comprometiendo unidades en la PAA susceptibles a cambios tal como lo recomienda Cisneros et al., 2018 en donde el cambio de uso de la tierra es una variable ideal para este tipo de análisis espaciales. G* analiza los valores de una unidad respecto a valores de unidades vecinas $y$ 

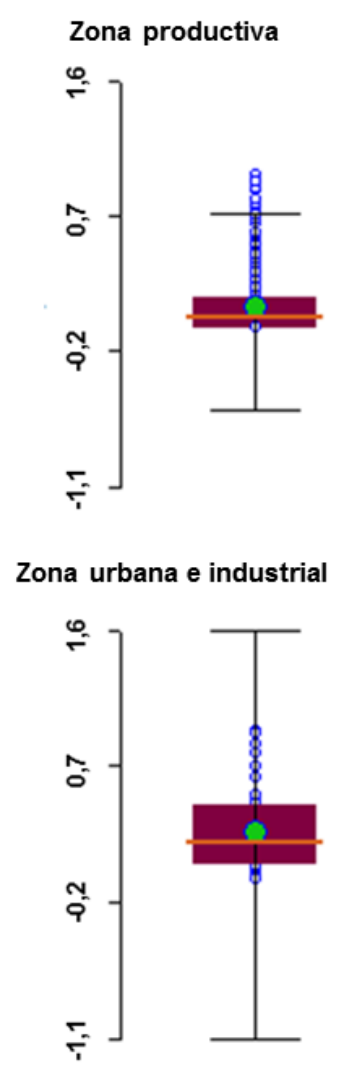

Zona de protección y conservación ecológica
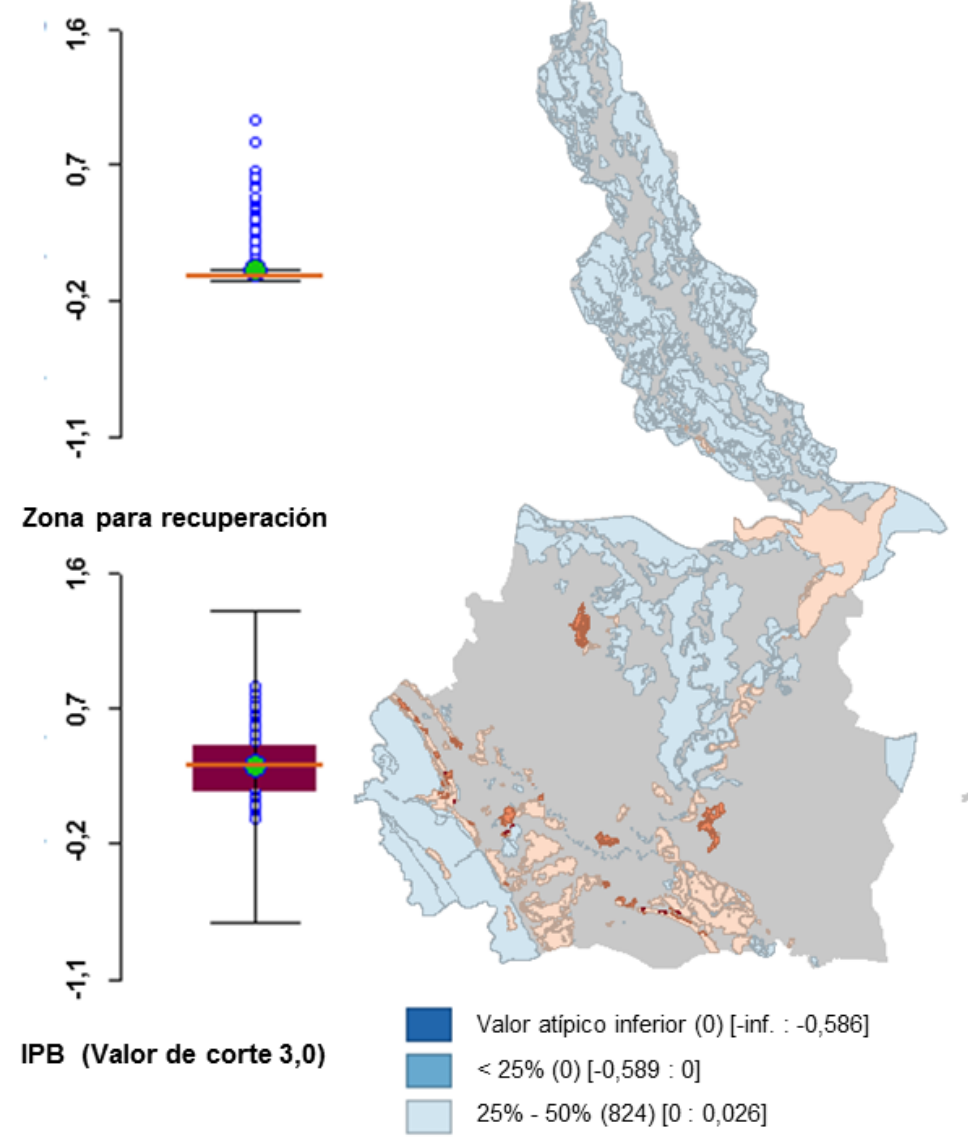

Zona productiva

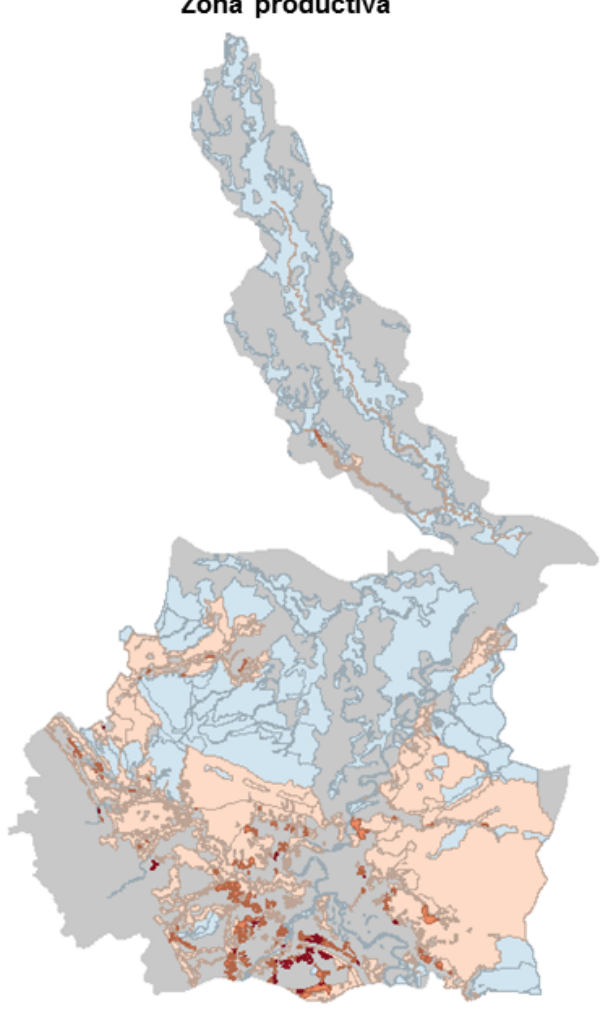

Zona de protección y conservación ecológica
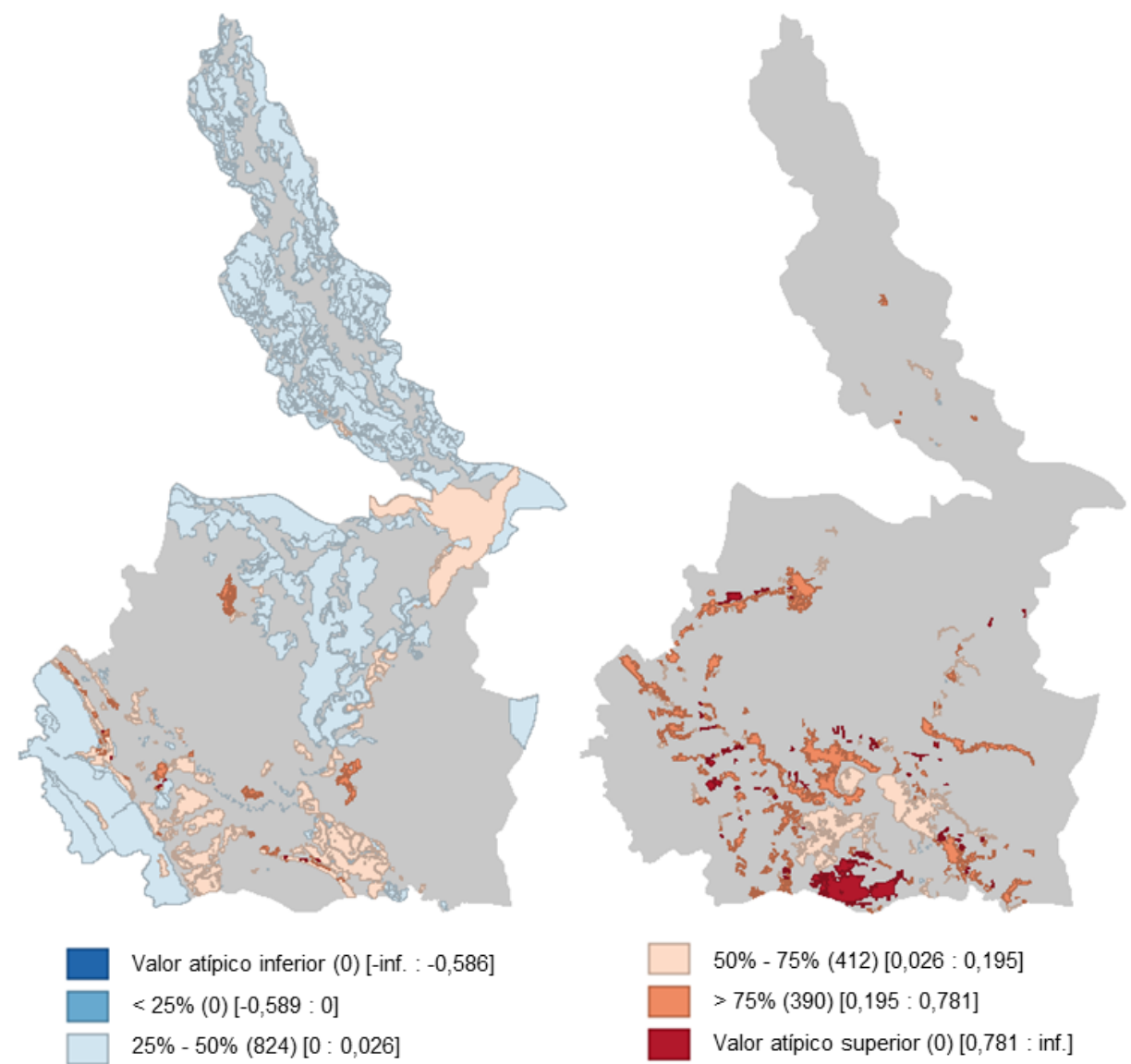

Figura 3. Distribución del índice de pérdida del bosque (IPB) por grandes zonas ecológicas económicas de la provincia Alto Amazonas, departamento Loreto, Perú. Elaborado en GeoDa 1.16 . 
Tabla 1. Superficie de patrones espaciales de puntos fríos y calientes por nivel de confianza de la provincia Alto Amazonas, Loreto, Perú.

\begin{tabular}{lrrr}
\hline $\begin{array}{c}\text { Patrón espacial y su } \\
\text { confianza }\end{array}$ & $\begin{array}{c}\text { Superficie de la PAA } \\
\text { (ha) }\end{array}$ & $\begin{array}{c}\text { Superficie de la PAA } \\
\text { (\%) }\end{array}$ & $\begin{array}{c}\text { Promedio de } \\
\text { IPB }\end{array}$ \\
\hline Puntos fríos 99\% & 301309,42 & $15,03 \%$ & $1,33 \%$ \\
Puntos fríos 95\% & 214904,23 & $10,72 \%$ & $0,90 \%$ \\
Puntos fríos 90\% & 110476,58 & $5,51 \%$ & $0,45 \%$ \\
Sin significancia & 1001884,41 & $49,99 \%$ & $9,64 \%$ \\
Puntos calientes 90\% & 7552,21 & $0,38 \%$ & $41,06 \%$ \\
Puntos calientes 95\% & 38960,29 & $1,94 \%$ & $44,02 \%$ \\
Puntos calientes 99\% & 329200,67 & $16,42 \%$ & $44,90 \%$ \\
\hline
\end{tabular}

totales, así cualquier afectación sobre el contexto haría variar de un agrupamiento de frío a caliente, tal como indican Falcón y Ramírez (2015), en donde los cambios en un territorio se representaron de mejor forma con G*.

Así también, los patrones espaciales de puntos calientes indican una alta distribución en los distritos de Yurimaguas y Teniente Cesar López Rojas. El patrón espacial de puntos fríos se distribuye en los distritos de Lagunas y Jeberos (Tabla 2).

En la Figura 5, se puede observar la distribución del G* al 99\% de confianza (color rojo), con respecto a cada una de las cuatro grandes zonas de la ZEE, se observa como la pérdida del bosque genera un patrón espacial de puntos calientes en la ZP agrupados en áreas cercanas a las carreteras, esto es consistente con lo propuesto por la ZEE e indica que la pérdida del bosque se viene dando en áreas pequeñas sobre tierras aptas para uso productivo (Escobedo y Torres, 2015); así mismo, el patrón de puntos fríos se concentra en ambas márgenes de los ríos Huallaga, Aypena, Pavayacu, Nucuray, en el centro y norte de la PAA; esto es consistente con la propuesta de ZEE, también podemos observar áreas con no significancia, esto debido a que algunas unidades afectadas por la pérdida del bosque son vecinas o adyacentes a unidades no afectadas (Falcón y Ramírez, 2015).

En la ZUI se observa como la pérdida de bosque genera un patrón de puntos calientes agrupa- dos en parches aislados al sur de la PAA, esto es consistente con los usos propuestos por la ZEE ya que estos lugares reúnen las mejores características para ser habitados o ampliar las áreas urbanas existentes. Estos resultados pudieran contribuir a realizar evaluaciones detalladas en las unidades de la ZUI, debido a la amplia aplicación de los índices locales de autocorrelación como por ejemplo en la fragmentación de la vegetación en zonas urbanas (Kowe et al., 2019; Kowe et al., 2020).

En la ZPCE se observa agrupamientos de puntos calientes al sur de la PAA colindantes con áreas de patrones de puntos calientes de zonas propuestas para producción, lo cual está influenciado por el paso de carreteras y la ampliación de la frontera agrícola. Así mismo, la concentración de puntos fríos va desde el centro hacía el norte de la PAA y sigue el mismo patrón que los puntos fríos en zonas productivas, lo cual puede estar indicando un no uso de la información de la ZEE e inadecuado uso de las tierras por cultivos de subsistencia, este último debido a la deforestación en la ZP adyacentes a la ZPCE, G* pudiera estar captando estos patrones y agrupándolos (Ren et al., 2020). La ZPCE, específicamente en el sector norte de la PAA, está conformada por ecosistemas como bosques pantanosos de llanura aluvial, bosques pantanosos de palmas densas, bosques pantanosos de palmas mixtas y herbazales pantanosos de la llanura aluvial (Encarnación et al., 2015), estos proveen de servicios ecosistémicos culturales importantes para la PAA. Sería importante 


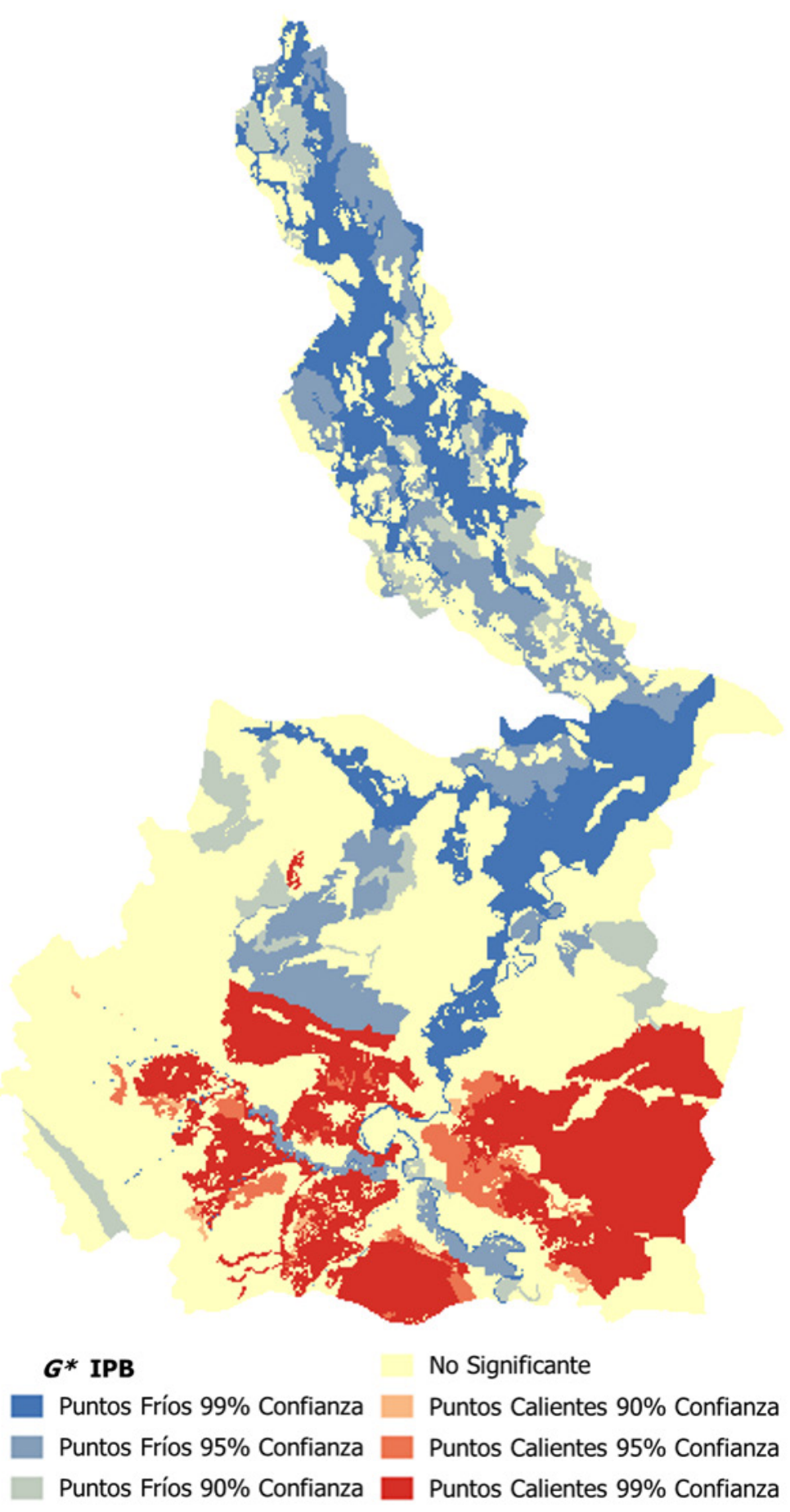

Figura 4. Mapa de patrones espaciales de puntos calientes y fríos de la pérdida del bosque aplicando el índice de Getis-Ord (G*) de la provincia Alto Amazonas, departamento Loreto, Perú. Elaborado en GeoDa 1.16. 
Tabla 2. Superficie expresada en porcentaje de patrones espaciales de puntos fríos y calientes por nivel de confianza de los distritos de la provincia Alto Amazonas, Loreto, Perú. PF (puntos fríos), SS (sin significancia) y PC (puntos calientes).

\begin{tabular}{lccccccc}
\hline \multirow{2}{*}{ Distritos de la PAA } & \multicolumn{7}{c}{ Superficie en \% por patrón espacial y confianza } \\
\cline { 2 - 8 } & PF 99\% & PF 95\% & PF 90\% & SS & PC 90\% & PC 95\% & PC 99\% \\
\hline Balsapuerto & 0,34 & 11,27 & 7,63 & 22,04 & 25,58 & 15,82 & 10,93 \\
Jeberos & 22,89 & 25,59 & 26,63 & 21,58 & 0,30 & 0,04 & 4,81 \\
Lagunas & 46,67 & 41,99 & 47,02 & 17,20 & 0,00 & 0,00 & 0,00 \\
Santa Cruz & 14,09 & 2,35 & 12,83 & 13,26 & 0,00 & 4,38 & 21,47 \\
Teniente César López Rojas & 1,59 & 3,43 & 3,52 & 4,70 & 23,30 & 32,54 & 24,72 \\
Yurimaguas & 14,42 & 15,36 & 2,37 & 21,23 & 50,82 & 47,23 & 38,07 \\
\hline
\end{tabular}

para una gestión adecuada del territorio continuar monitoreando los patrones que se generan en este sector y las características espaciales que estos configuran en el tiempo (Alvarado et al., 2019; Castro, 2017; Roces et al., 2017; Falcón y Ramírez, 2015), pudiendo ser complementado con índices espectrales (Otavo y Echevarrya, 2017), gradientes altitudinales (Zúñiga y Pompa, 2018), variables climáticas (Cisneros et al., 2019) o sociales, económicos y culturales (Plieninger et al., 2013 y Li et al., 2017) generando modelos cartográficos que contribuyan a un mejor análisis para la toma de decisiones en la gestión del territorio amazónico peruano.

En la ZR la pérdida del bosque se agrupa en patrones de puntos calientes al sur de la PAA, esto evidencia la pérdida de bosques secundarios en áreas que ya habían sido zonificadas principalmente por conflictos por el uso no concordante con su vocación natural, esta última representada por la Capacidad de Uso Mayor de la Tierra. Estos resultados refuerzan la hipótesis planteada, pero no se corresponden con lo propuesto por Álvarez y Jarama (2015), quienes ya habían identificado conflictos por sobre uso y sub uso de la tierra en la PAA y recomendaron una intervención estratégica para la recuperación de estas áreas en base a su vocación natural.

Los resultados indican que $\mathrm{G}^{*}$ tiene la capacidad de representar patrones espaciales de la distribución del IPB agrupados en puntos fríos y calientes estadísticamente significativos, al igual que en otros estudios en donde se apli- ca este enfoque geoespacial (Ren et al., 2020; Zúñiga et al., 2019; Alvarado et al., 2018; Elfadaly et al., 2018; Cisneros et al., 2018; Zúñiga y Pompa, 2018, Ord y Getis, 1995), esto permite comprender e inferir focos en donde se debe prestar atención a la dinámica de la deforestación en la PAA, así como concluyen Philippe y Karume (2019), y de acuerdo a Li et al. (2017) en donde un $\mathrm{p}<0,05$ o nivel de confianza del $95 \%$ se puede definir como significativo estadísticamente.

Se puede deducir que la ZEE desde su generación y aprobación no ha contribuido a frenar el avance de la deforestación, el mejor uso que se le puede dar a la ZEE es aplicarlo directa o indirectamente en la gestión del uso del territorio, minimizando así la pérdida de bosque en las zonas categorizadas para la conservación, protección y recuperación. Así tenemos algunos ejemplos que se han dado en la Amazonía peruana, de acuerdo a los usos propuestos en la ZEE Iquitos-Nauta (IIAP-AECI-CTARL, 2001; IIAP, 1996) se creó la Reserva Nacional Allpahuayo Mishana en la zona propuesta para la conservación (BIODAMAZ, 2007). Otro ejemplo positivo, fue el uso de la ZEE para la conservación en el departamento de San Martin (Rodríguez et al., 2009) en donde se crearon áreas para la conservación regional de los Bosques de Shunté y Mishollo - Boshumi (Augusto, 2018). En el departamento Amazonas se usaron los resultados de la ZEE para la creación de varias unidades de conservación, como el Área de Conservación Ambiental de la Cuenca del Río Huamanpata 

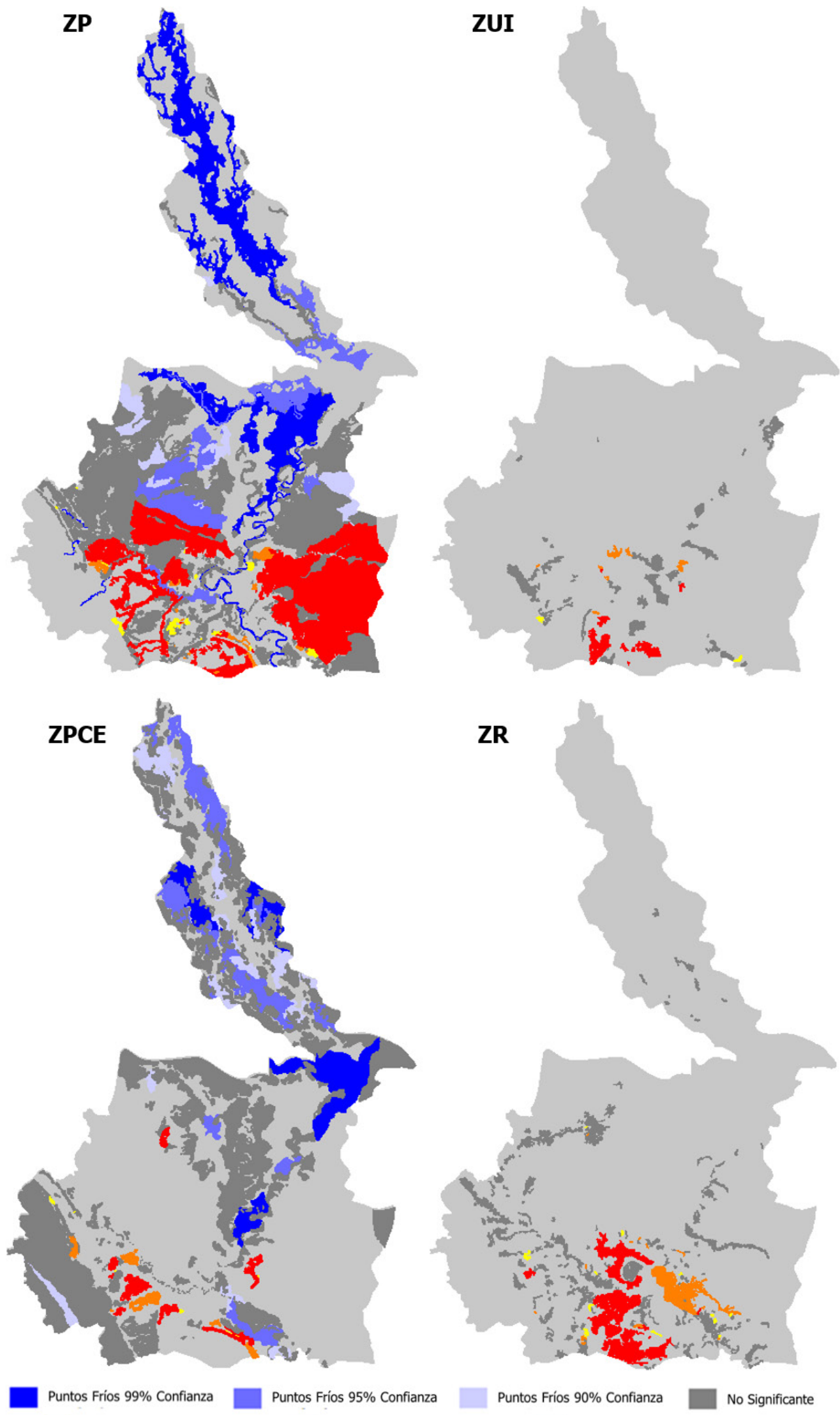

Puntos Calientes $90 \%$ Confianza

Puntos Calientes $95 \%$ Confianza

Puntos Calientes $99 \%$ Confianza

Figura 5. Distribución de patrones espaciales de puntos calientes y fríos por gran zona ecológica económica de la provincia Alto Amazonas, departamento Loreto, Perú. ZP (Zonas productivas), ZUI (Zonas urbano industriales), ZPCE (Zonas para Protección y conservación ecológica) y ZR (Zonas para recuperación). Elaborado en GeoDa 1.16. 
(Ordenanza Municipal No 002-2006-MPRM). Por el contrario, en otros lugares del departamento Amazonas no se utilizó la ZEE, desencadenando en una deforestación con notoria pérdida de bosques en zonas recomendadas para la protección y recuperación (IIAP-GRA, 2010; Zumaeta, 2021). Igualmente, la ZEE de Madre de Dios (GOREMAD-IIAP, 2008) no fue utilizada, al menos para el sector de Huepetue, en donde se desarrolló la deforestación con fin minero (en su mayoría ilegal) en tierras aptas para cultivos, esto último se pudo deducir a partir de la comparación entre el mapa de la ZEE y la información disponible en el GEOBOSQUES.

Entonces, los estudios de ZEE en algunos casos fueron usados para la gestión del territorio y en otros no, lo que evidencia una falta de decisión y apoyo político (entre otros factores) (Mendoza, 2005), para continuar con la implementación y aplicación de la ZEE y el ordenamiento territorial.

\section{CONCLUSIÓN}

La propuesta de la ZEE desde su generación y aprobación no ha sido tomada en cuenta para la gestión del territorio de la provincia Alto Amazonas, por ende, no ha contribuido a mitigar la pérdida del bosque por deforestación. De acuerdo a los resultados de la presente investigación, las grandes zonas ecológicas económicas más afectadas por la pérdida de bosque han sido aquellas que se propusieron para la recuperación por conflictos de uso. El conflicto de uso en este territorio viene en aumento debido a que tanto en las zonas propuestas para la protección y conservación ecológica, como en las de recuperación por conflictos de uso, se vienen observando patrones de concentración de una mayor superficie de pérdida del bosque, principalmente al sur de la provincia Alto Amazonas y en unidades de análisis próximas a otras con un fuerte cambio de uso por cultivos o expansión de la frontera agrícola, además de áreas colindantes a carreteras; mientras que una menor superficie de pérdida del bosque se concentra y distribuye en unidades de análisis alejadas, que no cuentan con vías de comunicación, siendo solamente accesibles por vía fluvial y en comunidades al norte de la provincia Alto Amazonas.

En base a los resultados obtenidos con la aplicación de la autocorrelación espacial local, se sugiere realizar estudios con un enfoque geoespacial a niveles más detallados de unidades ecológicas económicas, para análisis más puntuales entre el uso actual del territorio y las recomendaciones específicas para cada unidad de la ZEE. Con respecto a la aplicación o no de la ZEE, se recomienda generar e institucionalizar directivas indicando el proceso para el uso y aplicación de la información de la ZEE, así como de otros estudios similares, en la planificación estratégica, formulación, evaluación y ejecución de proyectos de inversión, entre otros. Así mismo, debido a la gran cantidad de información que se genera en un proceso de ZEE y la carencia de decisión política mencionada anteriormente, esperamos que el presente estudio contribuya, al diseño e implementación de sistemas en inteligencia territorial basados en el análisis espacial de la geoinformación, para la toma de decisiones sobre el territorio.

\section{AGRADECIMIENTO}

Al Programa Nacional para la Conservación de los Bosques del Ministerio del Ambiente por la disponibilidad de los datos. A Marin Pompa-García y Eloy Montes Galbán por sus observaciones al presente manuscrito.

\section{REFERENCIAS BIBLIOGRÁFICAS}

Alvarado, R., Pompa, M., Zúñiga, J., Jiménez, M. (2019) Spatial analysis of phenotypic variables in a clonal orchard of Pinus arizonica Engelm. in northern Mexico. Revista Chapingo serie ciencias forestales y del ambiente, 25(2): 185-199.

Álvarez, L., Jarama, R. (2015) Sub modelo de Conflictos de Uso. Zonificación Ecológica y Económica - ZEE de la provincia Alto Amazonas, departamento Loreto. Iquitos - Perú. 
Álvarez L. y Jarama, R. (2015) Temático de Uso Actual de la Tierra. Zonificación Ecológica y Económica - ZEE de la provincia Alto Amazonas, departamento Loreto. Iquitos - Perú.

Anselin, L. (2021) GeoDa (Spatial Statistical Program). The Encyclopedia of Research Methods in Criminology and Criminal Justice, 2, 839-841.

Anselin, L., Syabri, I., Kho, Y. (2010) GeoDa: an introduction to spatial data analysis. In Handbook of applied spatial analysis. 7389.

Augusto, M. (2018) Cuando el Estado y la sociedad confluyen: la construcción de la política territorial en San Martín (2002-2018). Debates en Sociología, (47): 9-39.

Ballón, E., Campana, A., Glave, M. (2015) Ordenamiento territorial: entre el entrampamiento normativo y la reterritorialización del capital. Perú hoy: hacia otro desarrollo DESCO, Lima, 153-176.

BIODAMAZ. (2007) Reserva Natural Allpahuayo Mishana, Una joya natural al lado de Iquitos. Perú-Finlandia: Proyecto Diversidad Biológica de la Amazonía Peruana. BIODAMAZ-IIAP.

Brown, G., Raymond, C. (2014) Methods for identifying land use conflict potential using participatory mapping. Landscape and Urban Planning, 122, 196-208.

Castro, K. (2017) Segurança hídrica urbana: morfologia urbana e indicadores de serviços ecossistêmicos, estudo de caso do Distrito Federal, Brasil.

Cisneros-González, D., Zúñiga-Vásquez, J., Pompa-García, M. (2018) Actividad del fuego en áreas forestales de México a partir de sensores remotos y su sensibilidad a la sequía. Madera y bosques, 24(3).

Decreto Supremo Nº 087-2004-PCM.- Aprueban Reglamento de Zonificación Ecológica Económica (ZEE).

de Moura, E., Procopiuck, M. (2020) GIS-based spatial analysis: basic sanitation services in Parana State, Southern Brazil. Environmental monitoring and assessment, 192(2): 1-13.

Elfadaly, A., Attia, W., Qelichi, M., Murgante, B., Lasaponara, R. (2018) Management of cul- tural heritage sites using remote sensing indices and spatial analysis techniques. Surveys in Geophysics, 39(6): 1347-1377. Encarnación, F., Zárate, R., Mori, T. (2015) Temático de Vegetación. Zonificación Ecológica y Económica - ZEE de la provincia Alto Amazonas, departamento Loreto. Iquitos - Perú.

Escobedo, R., Torres, G. (2015) Temático de Suelos y Capacidad de Uso Mayor de las tierras. Zonificación Ecológica y Económica - ZEE de la provincia Alto Amazonas, departamento Loreto. Iquitos - Perú.

Escobedo, R., Torres, G., Castro, W. (2015) Temático de Fisiografía. Zonificación Ecológica y Económica - ZEE de la Provincia Alto Amazonas, departamento Loreto. Iquitos - Perú.

Gobierno Regional de Loreto, Municipalidad Provincial Alto Amazonas e Instituto de Investigaciones de la Amazonía Peruana. (2015) Zonificación Ecológica y Económica - ZEE de la Provincia Alto Amazonas, departamento Loreto.

GOREMAD-IIAP. (2008) Zonificación Ecológica y Económica del Departamento de Madre de Dios.

IIAP-AECI-CTARL (2001) Zonificación Ecológica Económica del área de influencia de la carretera Iquitos - Nauta. Iquitos: IIAP-AECICTARL.

IIAP-MPDM (2009) Zonificación Ecológica y Económica de la cuenca Pastaza-Morona. Iquitos: Instituto de Investigaciones de la Amazonía Peruana y Municipalidad Provincial Datem del Marañon.

IIAP-GOREL-MPAM. (2015) Mesozonificación Ecológica y Económica de la Provincia Alto Amazonas.

IIAP (Instituto de Investigación de la Amazonia Peruana) y GRA (Gobierno Regional de Amazonas). (2010) Zonificación Ecológica Económica del departamento de Amazonas. Lima. Perú.

Kowe, P., Mutanga, O., Odindi, J., Dube, T. (2019) Exploring the spatial patterns of vegetation fragmentation using local spatial autocorrelation indices. Journal of Applied Remote Sensing, 13(2): 024523. 
Kowe, P., Mutanga, O., Odindi, J., Dube, T. (2020) A quantitative framework for analysing long term spatial clustering and vegetation fragmentation in an urban landscape using multi-temporal landsat data. International Journal of Applied Earth Observation and Geoinformation, 88, 102057.

Li, Y., Zhang, L., Yan, J., Wang, P., Hu, N., Cheng, W., Fu, B. (2017) Mapping the hotspots and coldspots of ecosystem services in conservation priority setting. Journal of Geographical Sciences, 27(6): 681-696.

Limachi, L., Grandez, R. (2015) Temático de Socioeconomía. Zonificación Ecológica y Económica - ZEE de la provincia Alto Amazonas, departamento Loreto. Iquitos - Perú.

Mamani, S., Servan, L. (2017) Evaluación multitemporal de la deforestación en el distrito de Molinopampa, Provincia de Chachapoyas, Departamento Amazonas, 2015.

Mendoza, M. (2005) Ordenamiento territorial y conservación de la diversidad biológica. Zonas áridas, 9(1): 149-160.

Mengatto, E., de Oliveira, R., Silva, J. (2017) O município de Marabá, PA frente ao ZEE na Amazônia Legal: avaliação das taxas de desmatamento. In Embrapa Informática Agropecuária-Artigo em anais de congresso (ALICE). In: SIMPÓSIO BRASILEIRO DE GEOGRAFIA FÍSICA APLICADA, 17; CONGRESSO NACIONAL DE GEOGRAFIA FÍSICA, 1., 2017, Campinas. Os desafios da geografia física na fronteira do conhecimento. Campinas: Unicamp, 2017.

Ministerio de Cultura. (2021) Base de datos de pueblos indígenas u originarios. https:// bdpi.cultura.gob.pe/.

Ministerio de Relaciones Exteriores. (1998) Manual de Zonificación Ecológica - Económica para la Amazonía Peruana. Comisión Nacional Permanente Peruana del Tratado de Cooperación Internacional. Lima, Perú.

Ocampo, C., Cucho, E., Linares, W., Lavalle, F. (2018) Planeamiento Estratégico de la Provincia de Alto Amazonas. Lima, Perú.

Ord, J., Getis, A. (1995) Local spatial autocorrelation statistics: distributional issues and an application. Geographical analysis, 27 (4): 286-306.
Otavo, S., Echeverría, C. (2017) Fragmentación progresiva y pérdida de hábitat de bosques naturales en uno de los hotspot mundiales de biodiversidad. Revista mexicana de biodiversidad, 88 (4): 924-935.

Paredes, M. (2015) Clima, informe temático. Proyecto Zonificación Ecológica y Económica de la provincia de Alto Amazonas. Instituto de Investigaciones de la Amazonía Peruana-BioCan. Iquitos, Perú.

Peng, J., Hu, X., Qiu, S., Meersmans, J., Liu, Y. (2019) Multifunctional landscapes identification and associated development zoning in mountainous area. Science of the Total Environment, 660, 765-775.

Philippe, M., Karume, K. (2019) Assessing Forest cover change and deforestation hotspots in the north Kivu Province, DR-Congo using remote sensing and GIS. Am J Geogr Inf Syst, 8(2): 39-54.

Plieninger, T., Dijks, S., Oteros-Rozas, E., Bieling, C. (2013) Assessing, mapping, and quantifying cultural ecosystem services at community level. Land use policy, 33, 118129.

Ren, H., Shang, Y., Zhang, S. (2020) Measuring the spatiotemporal variations of vegetation net primary productivity in Inner Mongolia using spatial autocorrelation. Ecological Indicators, 112, 106108.

Roces-Díaz, J., Burkhard, B., Kruse, M., Müller, F., Díaz-Varela, E., Álvarez-Álvarez, P. (2017) Use of ecosystem information derived from forest thematic maps for spatial analysis of ecosystem services in northwestern Spain. Landscape and Ecological Engineering, 13(1): 45-57.

Rodríguez, F., Limachi, L., Reátegui, F., Escobedo, R., Ramírez, J., Encarnación, F., Maco, J., Guzmán, W., Castro, W., Fachin, L., Martínez, P. (2009) Las potencialidades y limitaciones del departamento de San Martín: zonificación ecológica y económica del Departamento de San Martín. Gobierno Regional de San Martín, Instituto de Investigaciones de la Amazonía Peruana y Deutsche Gesellschaft für Technische Zusammenarbeit. Lima. 
Siabato, W., Manrique, J. (2019) La autocorrelación espacial y el desarrollo de la geografía cuantitativa. Cuadernos de Geografía. Revista Colombiana de Geografía, 28(1): 1-22.

Vargas, C., Montalban, J., Leon, A. (2019) Early warning tropical forest loss alerts in Peru using Landsat. Environmental Research Communications, 1(12): 121002.

Wei, S., Xiong, C., Luan, Q., Hu, Y. (2014) Protection zoning of arable land quality index based on local spatial autocorrelation. Transactions of the Chinese Society of Agricultural Engineering, 30(18): 249-256.

WWF-IIAP (2002) Propuesta preliminar de macrozonificación del Abanico del Pastaza. Iquitos: WWF-IIAP.

Zárate, R., Aquino, R., Palacios, J., Fachin, L., Paredes, P., Castro, W., Torres, G., Martínez, P., Maco, J., Rimachi, M. (2016) Áreas prioritarias para la conservación de la Provincia de Alto Amazonas, Loreto, Perú. Ciencia Amazónica (Iquitos), 6(2): 136-149.
Zumaeta, D. (2021) Análisis multitemporal y predictivo del cambio de uso del suelo y pérdida de cobertura vegetal. Revista Científica UNTRM: Ciencias Naturales e Ingeniería, 2(3): 81-89.

Zúñiga-Vásquez, J., Cisneros-González, D., Pompa-García, M. (2019) Drought regulates the burned forest areas in Mexico: the case of 2011, a record year. Geocarto International, 34(5): 560-573.

Zúñiga-Vásquez, J., Pompa-García, M. (2019) The occurrence of forest fires in Mexico presents an altitudinal tendency: a geospatial analysis. Natural Hazards, 96(1): 213-224.

\section{Conflictos de intereses}

Los autores declaramos que no tenemos conflicto de interés con la presente investigación y publicación. 